\title{
Interleukin-1 $\alpha$ drives the dysfunctional cross-talk of the airway epithelium and lung fibroblasts in COPD
}

\author{
Emmanuel T. Osei $i^{1,2,3}$, Jacobien A. Noordhoek ${ }^{1,2,4}$, Tillie L. Hackett ${ }^{3}$, \\ Anita I.R. Spanjer ${ }^{2,5}$, Dirkje S. Postma ${ }^{2,4}$, Wim Timens ${ }^{1,2}$, \\ Corry-Anke Brandsma ${ }^{1,2,6}$ and Irene H. Heijink ${ }^{1,2,4,6}$
}

\begin{abstract}
Affiliations: 'University of Groningen, University Medical Center Groningen, Dept of Pathology and Medical Biology, Groningen, The Netherlands. ${ }^{2}$ University of Groningen, University Medical Center Groningen, GRIAC Research Institute, Groningen, The Netherlands. ${ }^{3}$ University of British Columbia, Centre for Heart Lung Innovation, Dept of Anesthesiology, Pharmacology and Therapeutics, Vancouver, BC, Canada. "University of Groningen, University Medical Center Groningen, Dept of Pulmonology, Groningen, The Netherlands. ${ }^{5}$ University of Groningen, Dept of Molecular Pharmacology, Groningen, The Netherlands. ${ }^{6}$ These two authors contributed equally to this work.
\end{abstract}

Correspondence: Emmanuel T. Osei, Centre for Heart Lung Innovation, University of British Columbia and St Paul's Hospital, 1081 Burrard Street, Room 166, Burrard Building, Vancouver, BC V6Z 1Y6, Canada.

E-mail: Emmanuel.oseidahli.ubc.ca

ABSTRACT Chronic obstructive pulmonary disease (COPD) has been associated with aberrant epithelial-mesenchymal interactions resulting in inflammatory and remodelling processes. We developed a co-culture model using COPD and control-derived airway epithelial cells (AECs) and lung fibroblasts to understand the mediators that are involved in remodelling and inflammation in COPD.

AECs and fibroblasts obtained from COPD and control lung tissue were grown in co-culture with fetal lung fibroblast or human bronchial epithelial cell lines. mRNA and protein expression of inflammatory mediators, pro-fibrotic molecules and extracellular matrix (ECM) proteins were assessed.

Co-culture resulted in the release of pro-inflammatory mediators interleukin (IL)-8/CXCL8 and heat shock protein (Hsp70) from lung fibroblasts, and decreased expression of ECM molecules (e.g. collagen, decorin) that was not different between control and COPD-derived primary cells. This pro-inflammatory effect was mediated by epithelial-derived IL-1 $\alpha$ and increased upon epithelial exposure to cigarette smoke extract (CSE). When exposed to CSE, COPD-derived AECs elicited a stronger IL- $1 \alpha$ response compared with control-derived airway epithelium and this corresponded with a significantly enhanced IL- 8 release from lung fibroblasts.

We demonstrate that, through IL- $1 \alpha$ production, AECs induce a pro-inflammatory lung fibroblast phenotype that is further enhanced with CSE exposure in COPD, suggesting an aberrant epithelialfibroblast interaction in COPD.

@ERSpublications

IL-1 $\alpha$ regulates epithelial-fibroblast interaction, and may drive inflammation and tissue remodelling in COPD http://ow.ly/Rhy23008ytz

Editorial comment in: Eur Respir J 2016; 48: 301-304.

This article has supplementary material available from erj.ersjournals.com

Received: Nov 162015 | Accepted after revision: May 092016 | First published online: July 132016

Support statement: This study was funded by Stichting Astma Bestrijding. Funding information for this article has been deposited with FundRef.

Conflict of interest: Disclosures can be found alongside this article at erj.ersjournals.com

Copyright OERS 2016 


\section{Introduction}

Chronic obstructive pulmonary disease (COPD) is mainly caused by exposure to noxious particles, of which cigarette smoking is the major risk factor [1]. Presently, no cure exists for COPD, and current pharmacological treatments can only partly suppress symptoms and exacerbations [2]. The disease is characterised by chronic inflammation and defective tissue repair leading to irreversible chronic airflow limitation as a result of destruction of the gas-exchanging surface of the lung (emphysema), remodelling and narrowing of the small airways [2].

When inhaled, cigarette smoke first encounters the airway epithelium, which normally forms a continuous and highly regulated structural barrier that is part of the innate immune defence [3]. We have previously shown that cigarette smoke inhibits epithelial barrier function [4]. This damage can also cause the release of pro-inflammatory mediators (interleukin (IL)-8/CXCL8, IL-6) and danger signals known as damage-associated molecular patterns (DAMPS), such as IL-1 $\alpha$ and heat shock protein (Hsp70) [5]. Additionally, airway epithelium is a source of growth factors (e.g. transforming growth factor (TGF)- $\beta 1$ ) that can act on the underlying mesenchymal cells in the lamina propria to induce repair [6]. Mesenchymal fibrocytes, fibroblasts and smooth muscle cells are essential structural cells that produce various extracellular matrix (ECM) proteins within the lung, including collagens and decorin [7]. We have previously demonstrated decreased decorin production by primary lung fibroblasts from severe (Global Initiative for Chronic Obstructive Lung Disease (GOLD) 4) COPD patients compared with mild (GOLD 1) COPD patients [8]. Other groups have also demonstrated that lung fibroblasts respond to IL-1 $\beta$, IL-1 $\alpha$ [9] and prostaglandin PGE2 [10] stimulation by releasing IL-8/CXCL8 and IL-6, and specifically in the case of IL-1 $\beta$, by also downregulating their ECM protein production [11]. Epithelial-fibroblast communication has been shown to be involved in the pathogenesis of asthma $[12,13]$ and has also been proposed to contribute to idiopathic pulmonary fibrosis [14]. However, detailed knowledge on the direct interaction between airway epithelial cells (AECs) and the underlying pulmonary fibroblasts in COPD is limited, also with respect to the effects of cigarette smoke.

We hypothesise that dysfunctional epithelium-fibroblast communication through the release of mediators plays a key role in the chronic inflammation and remodelling processes in COPD, and that cigarette smoke exposure contributes to this aberrant process. The objective of the study was to develop a co-culture cell model to investigate the effect of cross-talk between AECs and lung fibroblasts from severe COPD patients and control subjects on pro-inflammatory mediator release and ECM expression. Furthermore, we investigated the role of cigarette smoke exposure on the cross-talk between AECs and fibroblasts in COPD.

\section{Materials and methods}

Human airway epithelial and lung fibroblasts

Human bronchial epithelial 16HBE14o- cells (kindly donated by Dr D.C. Gruenert, University of California, San Francisco, CA, USA) were cultured in Eagle's minimal essential medium (EMEM)/10\% fetal calf serum (FCS) as described previously [15]. Primary AECs were isolated as described previously [16] from tracheobronchial tissue of 13 COPD patients with severe disease undergoing lung transplantation and from leftover tracheobronchial tissue of 16 non-COPD control donor lungs, for whom no further information was available. Subject characteristics of the donors are given in table 1. Primary AECs were cultured in hormonally supplemented bronchial epithelium growth medium (Lonza, Basel, Switzerland) and used at passage 3 as described previously [15].

Fetal lung fibroblast cells (MRC-5; BioWhittaker, Walkersville, MD, USA) were cultured in EMEM/10\% FCS. Primary human lung fibroblasts (PHLFs) were derived from nine COPD patients with severe disease undergoing lung transplantation and five non-COPD controls undergoing tumour resection surgery. Fibroblasts were isolated from lung parenchyma using the explant technique as described previously [8, 17], grown in Ham's F12 medium/10\% FCS (Lonza, Basel, Switzerland) and used for experiments at passage 5. The full protocol of PHLF isolation can be found in the online supplementary material. Subject characteristics of PHLF donors are available in table 2. The study protocol was consistent with the Research Code of the University Medical Center Groningen (www.rug.nl/umcg/onderzoek/researchcode/ index), and national ethical and professional guidelines (www.federa.org).

\section{Co-culture model}

16HBE14o- and MRC-5 cells were initially used to develop the model. Significant observations were replicated using: 1) primary AECs from severe COPD patients or controls with MRC-5 fibroblasts and 2) 16HBE14o- cells with PHLFs from COPD patients or controls to assess disease-specific effects in each cell type separately. Briefly, AECs were plated on $0.4-\mu \mathrm{M}$ pore $6.5-\mathrm{mm}$ transwell membranes (Costar; Corning, New York, NY, USA) and fibroblasts were seeded on 24-well plates. When both cell layers were confluent, the transwell insert with AECs was placed in co-culture with the fibroblasts and left for $72 \mathrm{~h}$ in the appropriate medium (see online supplementary material). 
TABLE 1 Characteristics of severe chronic obstructive pulmonary disease patients from whom primary airway epithelial cells (AECs) were obtained

\begin{tabular}{lccccccc} 
Patient & Age years & Sex & Smoking status & Pack-years & FEV $\mathbf{\%}$ pred & FEV $/$ FVC $\%$ & Experiment \\
\hline $\mathbf{1}$ & 53 & Male & Ex & 40 & 25 & 25 & Co-culture and conditioned medium \\
$\mathbf{2}$ & 58 & Female & Ex & 38 & 60 & 46 & Co-culture \\
$\mathbf{3}$ & 57 & Male & Ex & 30 & 11 & 31 & Co-culture \\
$\mathbf{4}$ & 64 & Male & Never & 0 & 39 & 53 & Co-culture \\
$\mathbf{5}$ & 44 & Male & Ex & 25 & 60 & 50 & Co-culture \\
$\mathbf{6}$ & 60 & Male & Ex & 23 & 16 & 29 & Conditioned medium \\
$\mathbf{7}$ & 48 & Male & Ex & 25 & 17 & 21 & Conditioned medium \\
$\mathbf{8}$ & 57 & Female & Ex & 45 & 23 & 24 & Conditioned medium \\
$\mathbf{9}$ & 57 & Female & Ex & 40 & 18 & 25 & Conditioned medium \\
$\mathbf{1 0}$ & 61 & Female & Ex & 35 & 19 & 23 & Conditioned medium \\
$\mathbf{1 1}$ & 49 & Male & Ex & 11 & 20 & 22 & Conditioned medium \\
$\mathbf{1 2}$ & 55 & Female & Ex & 72 & 14 & 29 & Conditioned medium \\
$\mathbf{1 3}$ & 62 & Male & Ex & 44 & 22 & Conditioned medium \\
\hline
\end{tabular}

FEV1: forced expiratory volume in $1 \mathrm{~s}$; FVC: forced vital capacity. Primary AECs were either used for co-culture experiments with MRC-5 or conditioned medium experiments.

Conditioned medium experiments and neutralising antibody experiments

16HBE14o- cells or primary AECs when confluent were serum/hormone-deprived overnight and stimulated with or without $20 \%$ cigarette smoke extract (CSE) for $6 \mathrm{~h}$. The CSE was thoroughly washed off and cells were incubated for another $24 \mathrm{~h}$ prior to the CSE-free conditioned medium being collected. Fibroblasts that had been serum-deprived overnight were then treated for $24 \mathrm{~h}$ with the CSE-free conditioned medium that had been pre-incubated for $1 \mathrm{~h}$ with or without $4 \mu \mathrm{g} \cdot \mathrm{mL}^{-1} \mathrm{IL}-1 \alpha$ neutralising antibody (AB-200-NA) or IL-1 $\beta$ neutralising antibody (MAB601) (R\&D Systems, Europe, Abingdon, UK). Cell-free supernatants were collected and analysed by ELISA, and cell lysates were harvested for RNA and protein examination. Refer to the online supplementary material for full protocols of experiments.

\section{Statistics}

Data were analysed using SPSS (IBM, Armonk, NY, USA). The Mann-Whitney U-test was used for comparison between subject groups and the Wilcoxon signed-rank test for paired comparisons within groups of primary cells. We tested for normal distribution on the outcomes of the experiments with cell lines and used the $\mathrm{t}$-test for paired differences accordingly. $\mathrm{p}<0.05$ was considered to be statistically significant.

TABLE 2 Characteristics of control donors and severe chronic obstructive pulmonary disease patients from whom primary human lung fibroblasts (PHLFs) were obtained

\begin{tabular}{|c|c|c|c|c|c|c|}
\hline Patient & Age years & Sex & Smoking status & Pack-years & FEV $1 \%$ pred & FEV $1 /$ FVC $\%$ \\
\hline \multicolumn{7}{|c|}{ Control-derived PHLFs } \\
\hline 1 & 67 & Female & Never & 0 & 101 & 81 \\
\hline 2 & 65 & Female & Current & 38 & 98 & 76 \\
\hline 3 & 74 & Male & Ex & 50 & 100 & 71 \\
\hline 4 & 65 & Male & Ex & 40 & 97 & 76 \\
\hline 5 & 50 & Male & Ex & 31 & 97 & 78 \\
\hline \multicolumn{7}{|c|}{ COPD-derived PHLFs } \\
\hline 6 & 59 & Male & Ex & 38 & 19 & 25 \\
\hline 7 & 58 & Female & Ex & 30 & 18 & 28 \\
\hline 8 & 60 & Male & Ex & 30 & 37 & 49 \\
\hline 9 & 66 & Male & Ex & NA & 24 & 31 \\
\hline 10 & 62 & Male & Ex & 44 & 22 & 19 \\
\hline 11 & 57 & Female & Ex & 40 & 18 & 25 \\
\hline 12 & 48 & Male & Ex & 27 & 12 & 23 \\
\hline 13 & 57 & Female & Ex & 33 & 25 & 33 \\
\hline 14 & 44 & Male & Ex & 25 & 60 & 50 \\
\hline
\end{tabular}

FEV1: forced expiratory volume in $1 \mathrm{~s}$; FVC: forced vital capacity; NA: not available. 


\section{Results}

Increased inflammatory mediator release in lung fibroblasts when in co-culture with AECS

When 16HBE14o- and MRC-5 cells were placed in co-culture, we found a significant increase in basolateral IL-8/CXCL8 (figure 1a) and Hsp70 (figure 1b) secretion compared with epithelial and fibroblast mono-cultures. IL-1 $\beta$ protein levels were undetectable (data not shown). Subsequent mRNA analyses on the epithelial and fibroblast cell fractions demonstrated that fibroblasts are the main source of secreted IL-8/CXCL8 (figure 1c). Similarly, IL-1 $\beta$ mRNA levels were increased in fibroblasts, but not in epithelial cells, when placed in co-culture (figure 1d). To determine if the results obtained from the cell lines in co-culture were representative of primary cells, we paired the 16HBE14o- cells with PHLFs derived from COPD and control subjects, and also paired primary AECs from COPD and control donors with MRC-5 cells. As in the co-culture cell line model, we found that co-culture of PHLFs with 16HBE14ocells also induced a significant increase in basolateral IL-8/CXCL8 (figure 1e) and Hsp70 (figure 1f), without differences in the IL-8/CXCL8 response of PHLFs from ex-smokers, the never-smoker and the current smoker. Furthermore, we confirmed that the mRNA for IL-8/CXCL8 (figure 1g) and IL-1 $\beta$ (figure $1 \mathrm{~h}$ ) was only upregulated in the PHLFs with co-culture. Additionally, combining primary AECs with MRC-5 fibroblasts resulted in increased basolateral IL-8/CXCL8 and Hsp70 levels as well as a trend towards an increase of IL-6 protein levels (online supplementary figure 1), while levels of granulocyte-macrophage colony-stimulating factor and IL-33 were undetectable. Hsp70 and IL-6 levels correlated strongly with IL-8/CXCL8 release in co-culture (online supplementary figure 2). We found no significant difference in the release of mediators between control and COPD-derived AECs and PHLFs.

\section{Decreased expression of ECM molecules and pro-fibrotic proteins in lung fibroblasts in} co-culture with epithelial cells

In contrast to the increased pro-inflammatory response, co-culture of PHLFs from COPD and control subjects with $16 \mathrm{HBE} 14 \mathrm{o}$ - cells resulted in a significant downregulation in the mRNA expression of $\alpha$-smooth muscle actin ( $\alpha$-SMA) (figure $2 \mathrm{a}$ ), TGF- $\beta 1$ (figure $2 \mathrm{~b}$ ), and the ECM molecules decorin (figure $2 \mathrm{c}$ ), fibulin-5 (figure $2 \mathrm{~d}$ ), collagen-I $\alpha 1$ (figure 2e) and fibronectin (figure 2f) compared with mono-culture conditions. The downregulation of fibronectin and $\alpha$-SMA was confirmed on the protein level using Western blotting (figure $2 \mathrm{~g}$ and $\mathrm{h}$ ). Neither the baseline expression nor the decrease of these ECM and structural proteins upon co-culture was significantly different between COPD and control-derived PHLFs.

\section{Epithelium-derived IL-1 $\alpha$ is responsible for pro-inflammatory phenotype switch in lung fibroblasts}

Next, we used conditioned medium from 16HBE14o- cells to investigate whether the observed effects could be due to a soluble factor. Indeed, we observed that epithelial conditioned medium also induced an increase in IL-8/CXCL8 secretion by MRC-5 fibroblasts (figure 3a). Since IL-1 $\beta$ and PGE2 have been shown to induce similar phenotype switches in fibroblasts $[9,11]$, we first studied the effect of an IL- $1 \beta$ neutralising antibody (figure $3 \mathrm{~b}$ ) and inhibition of the downstream effect of PGE2 by the use of an adenylate cyclase inhibitor, MDL-12,330A hydrochloride (online supplementary figure 4). Both did not prevent the IL-8/CXCL8 release from MRC-5 cells. The other agonist of the IL-1R1 receptor is IL- $1 \alpha$. Interestingly, we found that the use of a neutralising antibody against IL- $1 \alpha$ completely abrogated IL- 8 / CXCL8 secretion by MRC-5 fibroblasts (figure 3c).

To determine if the results obtained using $16 \mathrm{HBE} 14 \mathrm{o}-$ conditioned medium were representative of primary cells, we conducted the same experiments using conditioned medium from primary AECs from COPD and control donors on MRC-5 fibroblasts. As shown in figure 4, neutralisation of IL-1 $\alpha$ also abrogated primary AEC conditioned medium-induced IL-8/CXCL8 secretion (figure 4a) as well as IL-8/CXCL8 and IL-1 $\beta$ mRNA expression (figure $4 \mathrm{~b}$ and $\mathrm{c}$ ) in MRC-5 fibroblasts.

Furthermore, we assessed the effect of the neutralising antibody in the co-culture model itself. As shown in figure $4 \mathrm{~d}$, the addition of IL- $1 \alpha$ neutralising antibody in the basal compartment also inhibited the basolateral release of IL-8/CXCL8 upon co-culture of primary AECs from COPD and control subjects with MRC-5. There was also a strong trend towards the downregulation of basolateral IL-6 release (online supplementary figure 1). Moreover, IL- $1 \alpha$ neutralisation in the co-culture also prevented the downregulation of TGF- $\beta 1$, decorin, fibulin-5 and collagen-I $\alpha 1$ mRNA expression in MRC-5 cells when co-cultured with primary AECs (figure 5).

\section{Cigarette smoke exposure increases IL-1 $\alpha$ expression in AECs and subsequent IL-8/CXCL8} production in lung fibroblasts

To evaluate if epithelial exposure to cigarette smoke alters communication with lung fibroblasts, we exposed fibroblasts to conditioned medium from 16HBE14o- cells pre-treated with CSE. Exposure of epithelial cells to CSE significantly increased the release of IL-1 $\alpha$ protein (figure $6 \mathrm{a}$ ) and mRNA (figure $6 \mathrm{~b}$ ) compared with basal levels. Subsequent exposure of MRC-5 fibroblasts to conditioned medium from CSE-treated 16HBE140- cells induced a significantly stronger increase in IL-8/CXCL8 production than stimulation with 

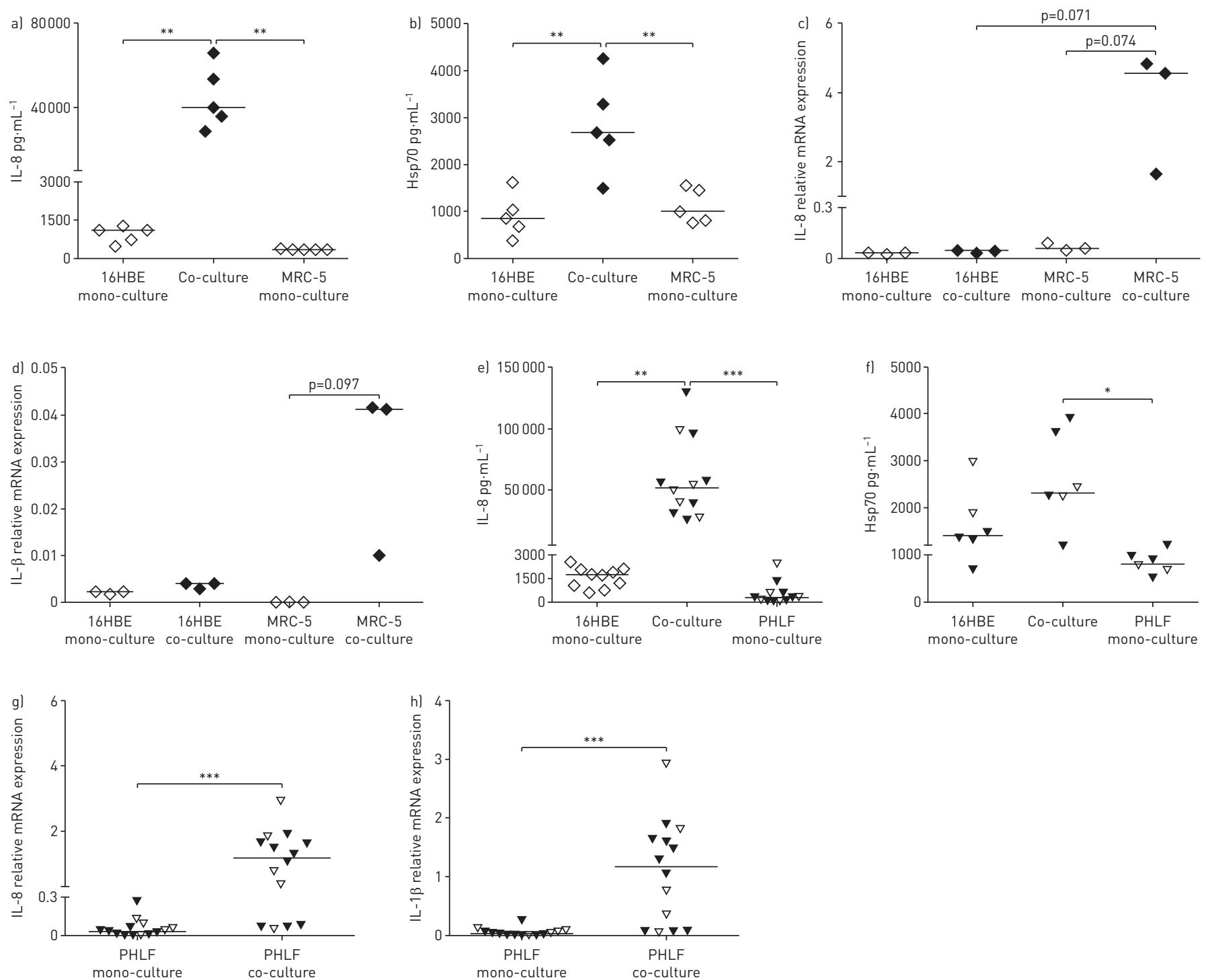

FIGURE 1 Interleukin (IL)-8/CXCL8, heat shock protein (Hsp70) and IL-1 $\beta$ levels in co-culture of airway epithelial cells (AECs) and lung fibroblasts. $16 \mathrm{HBE} 140-(16 \mathrm{HBE})$ cells were cultured alone and with MRC-5 cells or with primary human lung fibroblasts (PHLFs) derived from control (open triangles) and chronic obstructive pulmonary disease patients (filled triangles). a) IL-8/CXCL8 concentration (with median) and b) Hsp70 concentration (with median) in cell-free supernatants $(24 \mathrm{~h})$ of the basolateral compartment of co-culture system. C) IL-8/CXCL8 and d) IL-1 $\beta$ mRNA expression levels (with median) in epithelial cells and fibroblasts (6 h) harvested separately comparing co-culture and mono-cultures of 16HBE140- and MRC-5 cells. e) IL-8/CXCL8 concentration (with median) and f) Hsp70 concentration (with median) in cell-free supernatants (24 h) of the basolateral compartment. g) IL-8/CXCL8 and h) IL-1 $\beta$ mRNA expression levels (with median) in PHLFs (6 h) harvested separately comparing co-culture and mono-cultures of 16HBE140-cells and PHLFs. mRNA levels were related to the housekeeping genes $\beta_{2}$-microglobulin and protein phosphatase $1 \alpha$, and expressed as $2^{-\Delta C_{t}}{ }^{*}: p<0.05 ; * *: p<0.01 ;{ }^{* * *}: p<0.001$ between the indicated values.

basal 16HBE14o- conditioned medium (figure 6c). Similarly, CSE exposure significantly increased IL-1 $\alpha$ mRNA expression in our primary AECs (figure 6d).

Interestingly, COPD-derived primary AECs showed a stronger increase in IL- $1 \alpha$ mRNA expression after CSE exposure than control-derived primary AECs (figure 6d). In line with this increase in IL-1 $\alpha$ expression, conditioned medium from CSE-exposed COPD-derived primary AECs caused a significantly stronger increase in IL-8/CXCL8 release from MRC-5 fibroblasts than conditioned medium from CSE-exposed control primary AECs (figure 6e).

\section{Discussion}

We demonstrate that lung fibroblasts are directly regulated by AECs to release pro-inflammatory mediators and downregulate ECM synthesis and pro-fibrotic responses. Our data indicate that this regulation is driven by epithelium-derived IL- $1 \alpha$, as neutralising IL- $1 \alpha$ in epithelial conditioned media completely 

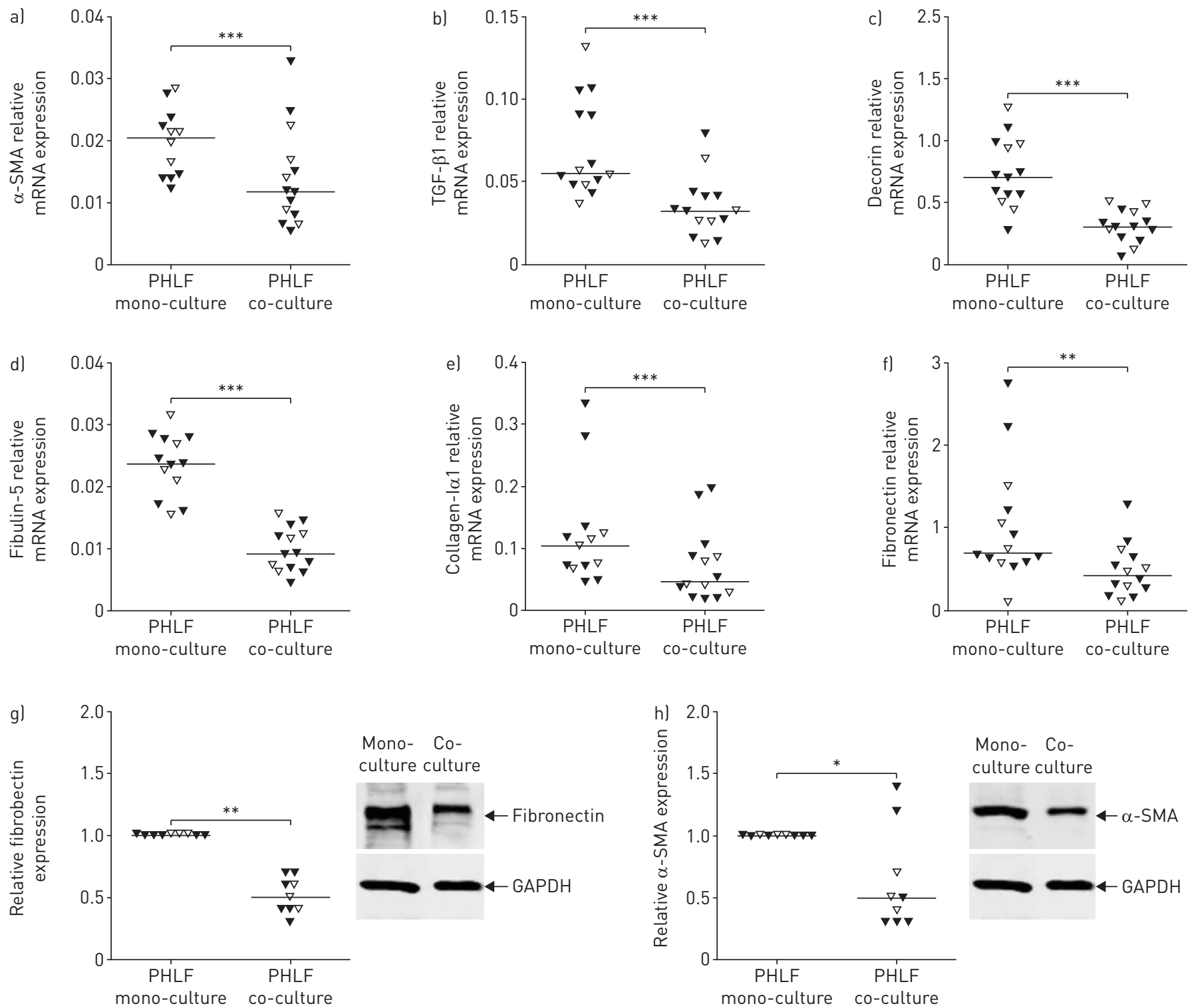

FIGURE 2 a-f) Decrease in the expression of extracellular matrix molecules and structural proteins in primary human lung fibroblasts (PHLFs) after co-culture with 16HBE140- cells. mRNA expression levels (6 h) (with median) of a) $\alpha$-smooth muscle actin ( $\alpha$-SMA), b) transforming growth factor (TGF)- $\beta 1, c$ ) decorin, d) fibulin-5, e) collagen-l $\alpha 1$ and f) fibronectin in PHLFs from control donors (open triangles) and chronic obstructive pulmonary disease (COPD) patients (filled triangles) comparing co-culture with 16HBE140- and mono-cultures. mRNA levels were related to the housekeeping genes $\beta_{2}$-microglobulin and protein phosphatase $1 \alpha$, and expressed as $2^{-\Delta C_{\mathrm{t}}} . \mathrm{g}, \mathrm{h}$ ) Relative protein expression levels (with median) and representative blots for g) fibronectin and h) $\alpha$-SMA in PHLFs from control donors (open triangles) and COPD patients (filled triangles) comparing co-culture with $16 \mathrm{HBE} 140-$ and mono-cultures. Glyceraldehyde 3-phosphate dehydrogenase (GAPDH) was used as loading control. *: $p<0.05 ;{ }^{* *}: p<0.01 ;{ }^{* * *}: p<0.001$ between the indicated values.

reversed the release of inflammatory mediators by lung fibroblasts. Additionally, we demonstrate that cigarette smoke exposure induces higher IL-1 $\alpha$ levels in AECs, particularly in epithelial cells from severe COPD patients. Moreover, cigarette smoke exposure may contribute to an aberrant cross-talk between epithelial cells and the underlying fibroblasts in COPD, as we observed that CSE-exposed epithelium derived from COPD patients induces a stronger increase in IL-8/CXCL8 secretion by lung fibroblasts than CSE-exposed control-derived epithelium.

In the lung, fibroblasts are located within the interstitium of the lung in close proximity to the airway epithelium, and hence can be easily influenced by the release of several factors by the epithelium in normal repair and disease states [7]. In COPD, however, the interaction between AECs and the underlying fibroblasts may be increased, and cells may be in closer contact by the observed fragmentation of the basement membrane in the mucosa $[18,19]$. Thus, the epithelium may exert stronger pro-inflammatory effects on fibroblasts in COPD. Fibroblasts in the lung have been shown to not only contribute to repair 

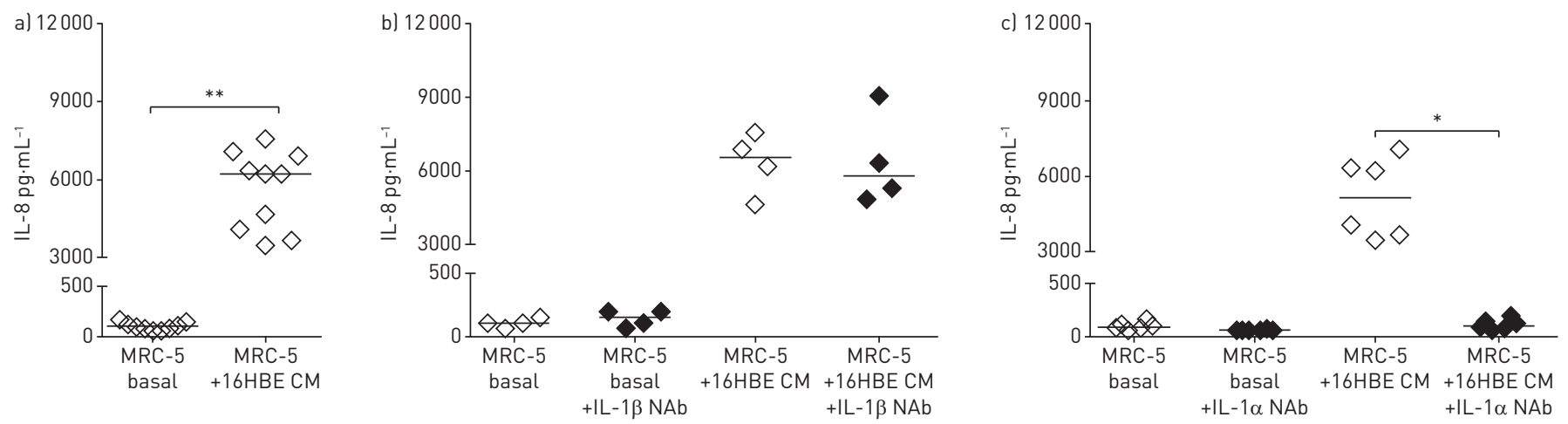

FIGURE 3 Interleukin (IL)-1 $\alpha$ from 16HBE140- cells is responsible for a pro-inflammatory phenotype switch in MRC-5 fibroblasts. IL-8/CXCL8 concentration (with median) released from confluent MRC-5 cells incubated a) without (MRC-5 basal) or with conditioned medium from confluent $16 \mathrm{HBE} 140$ - cells $(16 \mathrm{HBE} \mathrm{CM}), \mathrm{b})$ with $16 \mathrm{HBE} 140$ - conditioned medium in the presence and absence of $4 \mu \mathrm{g} \cdot \mathrm{mL}^{-1} \mathrm{IL}-1 \beta \mathrm{neutralising}$ antibody $(\mathrm{NAb})$ and c) with $16 \mathrm{HBE} 140-$ conditioned medium in the presence and absence of $4 \mu \mathrm{g} \cdot \mathrm{mL}^{-1} \mathrm{IL}-1 \alpha$ NAb. ${ }^{*}: \mathrm{p}<0.05$; ${ }^{* *}: \mathrm{p}<0.01$ between the indicated values.

processes through contraction, synthesis and remodelling of granulation tissue, but also through the production of cytokines to aid the normal immune defence mechanisms within the lung [20]. IL-8/CXCL8 is a chemoattractant for neutrophils in the lungs [21, 22], and chronic neutrophilic inflammation may contribute to abnormal tissue repair, remodelling and destruction in COPD [1]. Increased IL-8/CXCL8 release in the lungs has been associated with the pathogenesis of COPD [23]. Thus, higher IL-8/CXCL8 secretion by lung fibroblasts upon epithelial exposure to cigarette smoke may play a role in the
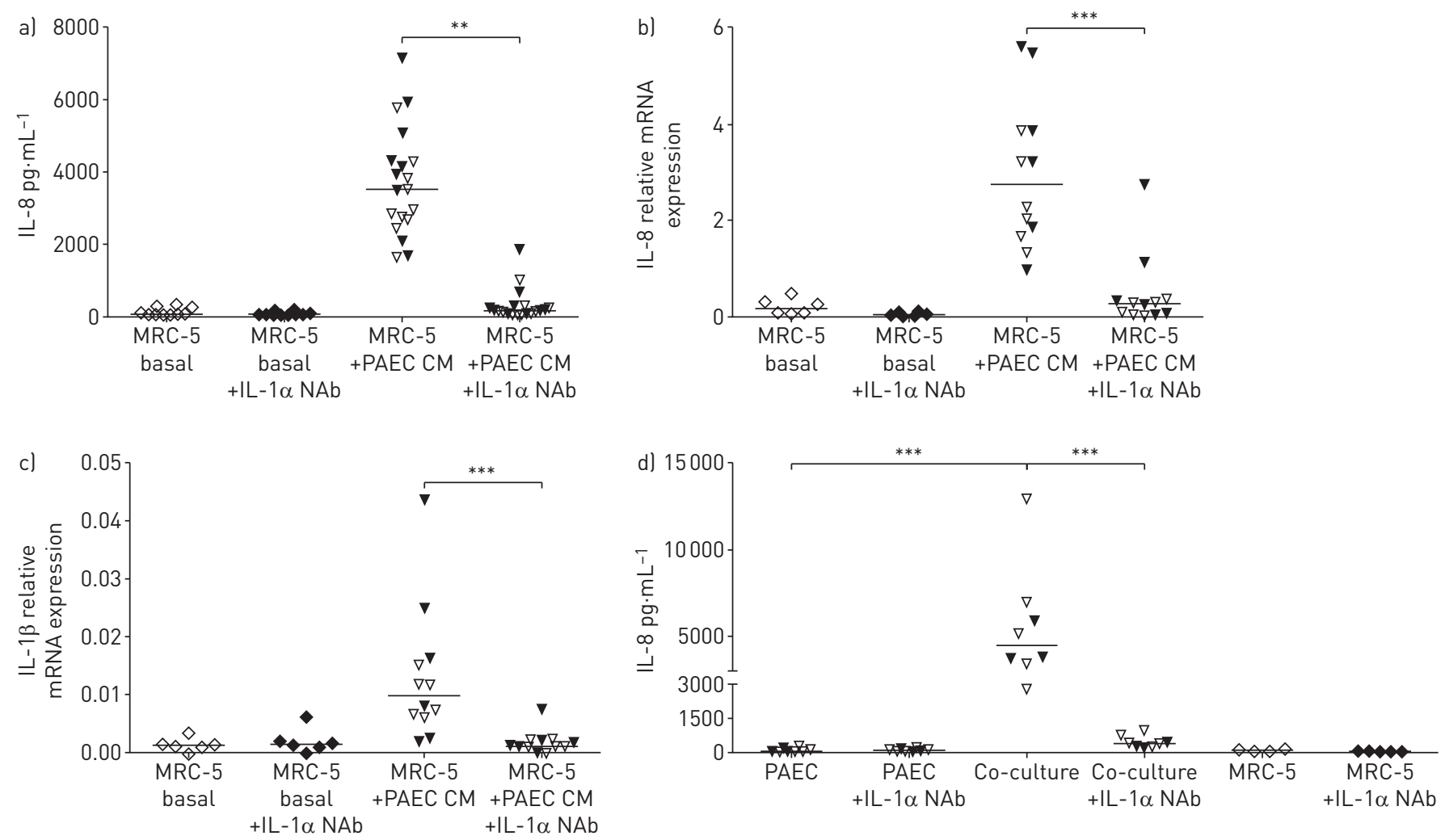

FIGURE 4 Interelukin (IL)-1 $\alpha$ from primary airway epithelial cells (AECs) is responsible for a pro-inflammatory phenotype switch in MRC-5 fibroblasts. MRC-5 cells were grown to confluence, serum-deprived overnight and subsequently incubated without (MRC-5 basal) or with conditioned medium from primary AECs (PAEC CM) of control donors lopen triangles) and chronic obstructive pulmonary disease (COPD) patients (filled triangles) in the presence or absence of $4 \mu \mathrm{g} \cdot \mathrm{mL}^{-1} \mathrm{IL}-1 \alpha$ neutralising antibody (NAb). a) IL-8/CXCL8 concentration (with median), and b) IL-8/CXCL8 and c) IL-1 $\beta$ mRNA expression (with median) in MRC-5 cells. d) IL-8/CXCL8 concentration (with median) in cell-free supernatants $(24 \mathrm{~h})$ of the basolateral compartment comparing co-culture and mono-cultures of primary AECs from control donors (open triangles) and COPD patients (filled triangles) and MRC- 5 cells in the presence or absence of $4 \mu \mathrm{g} \cdot \mathrm{mL}^{-1} \mathrm{IL}-1 \alpha$ NAb. mRNA levels were related to the housekeeping genes $\beta_{2}$-microglobulin and protein phosphatase $1 \alpha$, and expressed as $2^{-\Delta C_{t}}{ }^{* *}: p<0.01 ;{ }^{* * *}: p<0.001$ between the indicated values. 

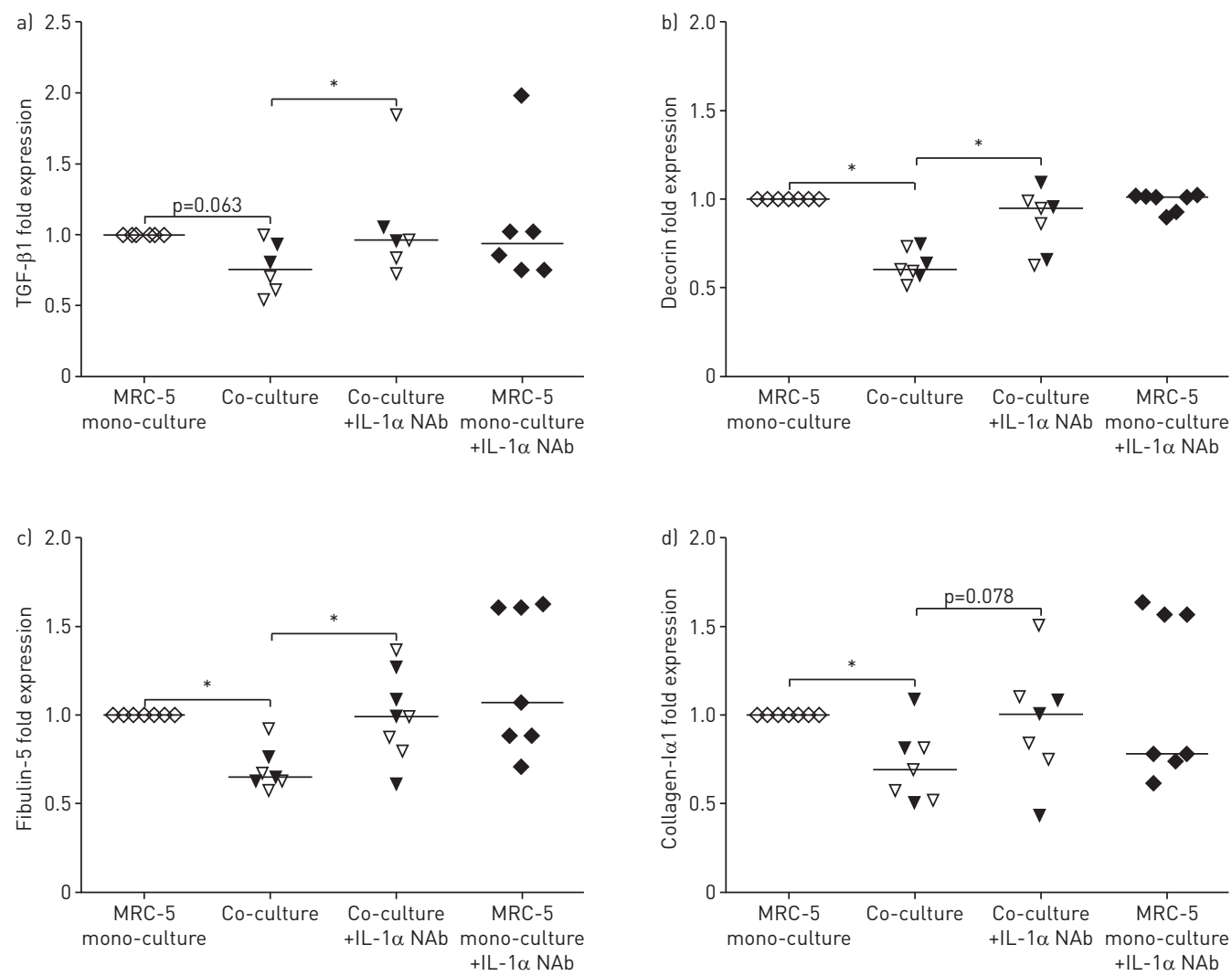

FIGURE 5 Interleukin (IL)-1 $\alpha$ is responsible for the decrease in expression of extracellular matrix molecules and structural proteins of lung fibroblasts after co-culture with epithelial cells. mRNA expression levels (with median) of a) transforming growth factor (TGF)- $\beta 1$, b) decorin, c) fibulin-5 and d) collagen-I $\alpha 1$ in MRC- 5 cells comparing mono-culture and co-culture with primary airway epithelial cells from control donors lopen triangles) and chronic obstructive pulmonary disease patients (filled triangles) in the presence and absence of $4 \mu \mathrm{g} \cdot \mathrm{mL}^{-1} \mathrm{IL}-1 \alpha$ neutralising antibody (NAb). mRNA levels were related to the housekeeping genes $\beta_{2}$-microglobulin and protein phosphatase $1 \alpha$, and expressed as $2^{-\Delta C_{t}}{ }^{*}: p<0.05$ between the indicated values.

pathogenesis of COPD [1]. Similarly, the increased release of Hsp70 and IL-6, which are both inflammatory mediators, has been implicated in chronic inflammatory processes in COPD $[5,24]$.

The airway epithelium has been reported to be the source of mediators that drive chronic inflammation and structural changes in COPD. Among others, IL- $1 \beta$, IL- $1 \alpha$, PGE2, tumour necrosis factor- $\alpha$, IL- 6 and various matrix metalloproteinases are increased in pulmonary epithelial cell supernatants from COPD patients compared with healthy controls [25]. PGE2 and IL-1 $\beta$ as well as IL-1 $\alpha$ have been shown to induce an increase in IL-8/CXCL8 secretion from fibroblasts $[9,10]$. Our results highlight that neutralisation of IL-1 $\alpha$ completely blocked the production of IL-8/CXCL8 by lung fibroblasts upon stimulation with epithelial conditioned media. Thus, our data provide strong support for a role of epithelium-derived IL- $1 \alpha$ in the cross-talk between epithelial cells and fibroblasts during both normal immune defence in the lungs and aberrant repair in COPD. IL- $1 \alpha$ is a member of the IL- 1 superfamily of inflammatory cytokines. It plays a crucial role in normal immune responses in vivo and is constitutively expressed in lung epithelium [26]. IL- $1 \alpha$ and its agonist IL-1 $\beta$ bind to the IL-1R1 receptor and illicit a similar downstream response with the subsequent activation of transcription factors such as nuclear factor- $\kappa \mathrm{B}$ and activator protein-1 [26]. While the activity of IL-1 $\beta$ is dependent on the activation of the NLRP3 inflammasome and subsequent cleavage by caspase-1, IL-1 $\alpha$ is both active in its pro-form and upon cleavage by caspases [27].

Our findings suggest that cigarette smoke may increase the release of IL-1 $\alpha$ from the airway epithelium in severe COPD, which subsequently causes an additional increase in IL-8/CXCL8 release from fibroblasts, contributing to neutrophilic inflammation in vivo. Relatively little work has been done on the role of IL-1 $\alpha$ in COPD and our data suggest the need for a closer look at the role of IL-1 $\alpha$ in the pathogenesis of COPD. In line with our findings, PAUwels et al. [27] showed that cigarette smoke-induced inflammation in mice is dependent on the IL-1R1 receptor, and both IL- $1 \alpha$ and its agonist IL- $1 \beta$ are involved in neutrophilic inflammation. Furthermore, Вотецно et al. [28] showed that smoke-induced neutrophilic inflammation in mice was dependent on IL- $1 \alpha$, but not IL-1 $\beta$. They also measured IL- $1 \alpha$ protein in 

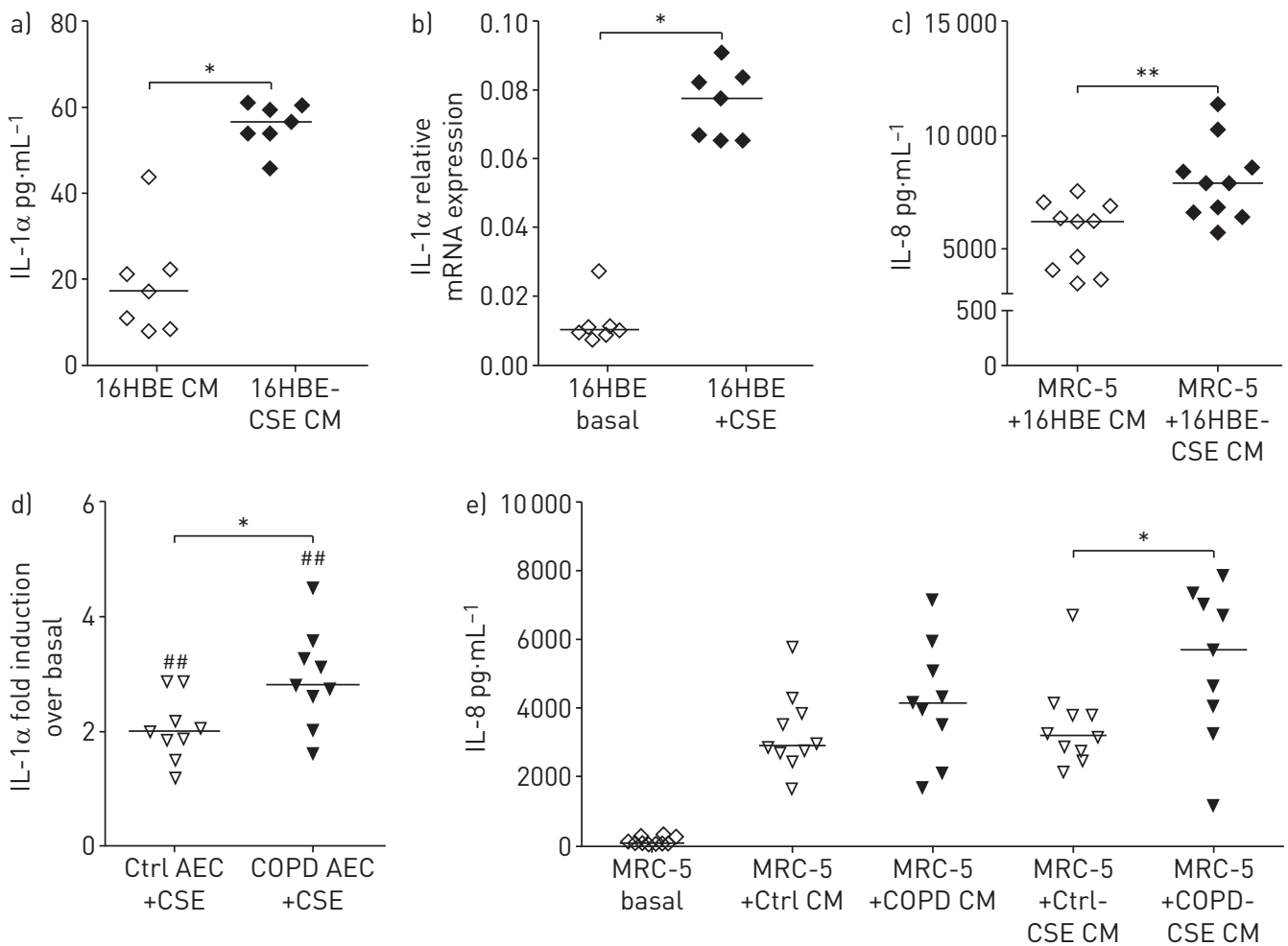

FIGURE 6 Interleukin (IL)-1 $\alpha$ from cigarette smoke extract (CSE)-exposed airway epithelium causes a higher release of IL-8/CXCL8 in lung fibroblasts. a-c)16HBE140- cells were pre-stimulated without or with $20 \%$ CSE. a) IL-1 $\alpha$ concentration (with median) in conditioned medium (16HBE CM) and b) IL-1 $\alpha$ mRNA expression (with median) in 16HBE140- cells. c) IL-8/CXCL8 concentration (with median) released from MRC-5 cells after incubation with 16HBE140- conditioned medium. d, e) Primary airway epithelial cells (AECs) from control (Ctrl) donors (open triangles) and chronic obstructive pulmonary disease (COPD) patients (filled triangles) were pre-stimulated with $20 \%$ CSE or not. d) Fold expression of IL- $1 \alpha$ in primary AECs. e) IL-8/CXCL8 concentration released from MRC-5 cells after incubation with conditioned medium from primary AECs. mRNA levels were related to the housekeeping genes $\beta_{2}$-microglobulin and protein phosphatase $1 \alpha$, and expressed as $2^{-\Delta C_{t}}$. ${ }^{*}: p<0.05 ;^{* *}: p<0.01$ for indicated values between groups; ${ }^{\# \#}: p<0.01$ for indicated values within groups.

bronchial biopsies and sputum, and showed an increased expression in the inflammatory infiltrate and epithelium in biopsies from COPD patients compared with controls [28]. SuwARA et al. [9] used another model of COPD where human AECs were injured in vitro with hydrogen peroxide and thapsigargin to induce reactive oxidative species injury and endoplasmic reticulum stress, respectively. They found increased levels of IL- $1 \alpha$ and IL-1 $\beta$ in the conditioned medium from these cells, which induced the release of IL-8/CXCL8 and IL-6 in MRC-5 fibroblasts [9]. This effect was completely blocked using IL-1 $\alpha$ neutralising antibody and only partially by using the IL-1 $\beta$ neutralising antibody. This is in line with our study, demonstrating a crucial role for epithelium-derived IL-1 $\alpha$ in regulating fibroblasts to become pro-inflammatory in a co-culture model representative of in vivo conditions. Our data further indicate that COPD-derived epithelial cells are more prone to release IL-1 $\alpha$ upon CSE exposure. It will be worthwhile to further study the mechanism of IL- $1 \alpha$ release in order to understand why COPD epithelial cells express more IL-1 $\alpha$ upon CSE exposure, e.g. whether this involves endoplasmic reticulum stress or an oxidant/ antioxidant imbalance as shown previously [9].

In addition to the production of inflammatory mediators in our co-culture model, AECs via IL-1 $\alpha$ also reduced the expression of the pro-fibrotic cytokine TGF- $\beta 1$, the structural molecule $\alpha$-SMA and various ECM molecules, including decorin, fibulin-5 and collagen-I 1 . The exact meaning of this finding needs to be investigated further, as the lack of demonstrating differences between COPD and control fibroblasts could also be due to power limitation. Nevertheless, our current findings support a mechanism whereby small airways may be lost due to defective tissue repair, which would be in line with the findings of McDonougH et al. [29], who demonstrated up to $90 \%$ loss of small airways in end-stage (GOLD 4) COPD patients. Although our epithelium-fibroblast model reflects the in vivo situation more closely than mono-cultures, our model may not fully reflect the in vivo situation, where other cell types are present as well. Also in our model, we compared control-derived primary AECs from first-generation tracheobronchial tissue to COPD-derived primary AECs from the third to fifth generation of the bronchial tree. Although these cells 
are not from the exact same site in the lung, various studies have shown similar genomic and epigenomic similarities in AECs from the tracheal and bronchial origin [30, 31]. We used cells derived from lung tissue of COPD patients undergoing transplantation for end-stage disease. Further experiments will be required to assess if the observed aberrant cross-talk between epithelial cells and fibroblasts is also present in early, mild and moderate stages of the disease. Future work will need to assess if the increased IL-8/CXCL8 secretion observed in our model additionally promotes neutrophil chemotaxis.

In conclusion, our data show that lung fibroblasts are regulated by AECs to become pro-inflammatory in their function. This regulation is driven by epithelial-derived IL-1 $\alpha$, further enhanced by CSE exposure and stronger in epithelial cells from severe COPD patients than from healthy individuals. Our study offers novel insights into the role of epithelial cells and fibroblasts in the pathogenesis of chronic remodelling and inflammation seen in COPD.

\section{References}

1 Hogg JC, Timens W. The pathology of chronic obstructive pulmonary disease. Annu Rev Pathol 2009; 4: 435-459.

2 Cosio MG, Saetta M, Agusti A. Immunologic aspects of chronic obstructive pulmonary disease. $N$ Engl J Med 2009; 360: 2445-2454.

3 Nyunoya T, Mebratu Y, Contreras A, et al. Molecular processes that drive cigarette smoke-induced epithelial cell fate of the lung. Am J Respir Cell Mol Biol 2014; 50: 471-482.

4 Heijink IH, Brandenburg SM, Postma DS, et al. Cigarette smoke impairs airway epithelial barrier function and cell-cell contact recovery. Eur Respir J 2012; 39: 419-428.

5 Pouwels SD, Heijink IH, ten Hacken NH, et al. DAMPs activating innate and adaptive immune responses in COPD. Mucosal Immunol 2014; 7: 215-226.

6 Araya J, Cambier S, Markovics JA, et al. Squamous metaplasia amplifies pathologic epithelial-mesenchymal interactions in COPD patients. J Clin Invest 2007; 117: 3551-3562.

7 Murray AL, Darryl AK, Laurent J. Fibroblast. In: Barnes PJ, Drazen JM, Rennard SI, et al., eds. Asthma and COPD: Basic Mechanisms and Clinical Management. Academic Press, San Diego, 2009; pp. 193-200.

8 Noordhoek JA, Postma DS, Chong LL, et al. Different modulation of decorin production by lung fibroblasts from patients with mild and severe emphysema. COPD 2005; 2: 17-25.

9 Suwara MI, Green NJ, Borthwick LA, et al. IL-1alpha released from damaged epithelial cells is sufficient and essential to trigger inflammatory responses in human lung fibroblasts. Mucosal Immunol 2014; 7: 684-693.

10 Van Ly D, Burgess JK, Brock TG, et al. Prostaglandins but not leukotrienes alter extracellular matrix protein deposition and cytokine release in primary human airway smooth muscle cells and fibroblasts. Am J Physiol Lung Cell Mol Physiol 2012; 303: L239-L250.

11 Mia MM, Boersema M, Bank RA. Interleukin-1beta attenuates myofibroblast formation and extracellular matrix production in dermal and lung fibroblasts exposed to transforming growth factor-beta1. PLoS One 2014; 9: e91559.

12 Reeves SR, Kolstad T, Lien TY, et al. Fibroblast-myofibroblast transition is differentially regulated by bronchial epithelial cells from asthmatic children. Respir Res 2015; 16: 21.

13 Malavia NK, Mih JD, Raub CB, et al. IL-13 induces a bronchial epithelial phenotype that is profibrotic. Respir Res 2008; 9: 27.

14 Prasad S, Hogaboam CM, Jarai G. Deficient repair response of IPF fibroblasts in a co-culture model of epithelial injury and repair. Fibrogenesis Tissue Repair 2014; 7: 7.

15 Heijink IH, Kies PM, Kauffman HF, et al. Down-regulation of E-cadherin in human bronchial epithelial cells leads to epidermal growth factor receptor-dependent Th2 cell-promoting activity. J Immunol 2007; 178: 7678-7685.

16 You Y, Richer EJ, Huang T, et al. Growth and differentiation of mouse tracheal epithelial cells: selection of a proliferative population. Am J Physiol Lung Cell Mol Physiol 2002; 283: L1315-L1321.

17 Brandsma CA, Timens W, Jonker MR, et al. Differential effects of fluticasone on extracellular matrix production by airway and parenchymal fibroblasts in severe COPD. Am J Physiol Lung Cell Mol Physiol 2013; 305: L582-L589.

18 Sohal SS, Reid D, Soltani A, et al. Reticular basement membrane fragmentation and potential epithelial mesenchymal transition is exaggerated in the airways of smokers with chronic obstructive pulmonary disease. Respirology 2010; 15: 930-938.

19 Gohy ST, Hupin C, Fregimilicka C, et al. Imprinting of the COPD airway epithelium for dedifferentiation and mesenchymal transition. Eur Respir J 2015; 45: 1258-1272.

20 Jordana M, Sarnstrand B, Sime PJ, et al. Immune-inflammatory functions of fibroblasts. Eur Respir J 1994; 7 : 2212-2222.

21 Martin TR, Frevert CW. Innate immunity in the lungs. Proc Am Thorac Soc 2005; 2: 403-411.

22 Keatings VM, Collins PD, Scott DM, et al. Differences in interleukin-8 and tumor necrosis factor-alpha in induced sputum from patients with chronic obstructive pulmonary disease or asthma. Am J Respir Crit Care Med 1996; 153: 530-534

23 Baines KJ, Simpson JL, Gibson PG. Innate immune responses are increased in chronic obstructive pulmonary disease. PLoS One 2011; 6: e18426.

24 Ferrari R, Tanni SE, Caram LM, et al. Three-year follow-up of interleukin 6 and C-reactive protein in chronic obstructive pulmonary disease. Respir Res 2013; 14: 24.

25 Holgate ST, Davies DE, Lackie PM, et al. Epithelial-mesenchymal interactions in the pathogenesis of asthma. J Allergy Clin Immunol 2000; 105: 193-204.

26 Garlanda C, Dinarello CA, Mantovani A. The interleukin-1 family: back to the future. Immunity 2013; 39 : 1003-1018.

27 Pauwels NS, Bracke KR, Dupont LL, et al. Role of IL-1alpha and the Nlrp3/caspase-1/IL-1beta axis in cigarette smoke-induced pulmonary inflammation and COPD. Eur Respir J 2011; 38: 1019-1028. 
28 Botelho MF, Bauer CMT, Finch D, et al. IL-1 alpha/IL1-R1 expression in chronic obstructive pulmonary disease and mechanistic relevance to smoke-induced neutrophilia in mice. PLoS One 2011; 6: e28457-e28457.

29 McDonough JE, Yuan R, Suzuki M, et al. Small-airway obstruction and emphysema in chronic obstructive pulmonary disease. N Engl J Med 2011; 365: 1567-1575.

30 Steiling K, Ryan J, Brody JS, et al. The field of tissue injury in the lung and airway. Cancer Prev Res (Phila) 2008; 1: 396-403.

31 Pezzulo AA, Starner TD, Scheetz TE, et al. The air-liquid interface and use of primary cell cultures are important to recapitulate the transcriptional profile of in vivo airway epithelia. Am J Physiol Lung Cell Mol Physiol 2011; 300: L25-L31. 